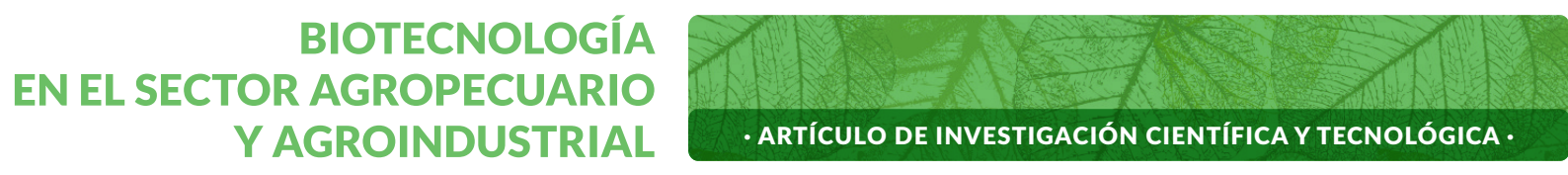

Vol. 18 No 2 · Julio - Diciembre 2020 • ISSN - 1692-3561 · ISSN-e 1909-9959 · DOI: http://dx.doi.org/10.18684

\title{
Análisis composicional de la pupa de gusano de seda (Bombyx mori L.)*
}

\section{Compositional analysis of silk worm pupae (Bombyx mori L.)}

\section{Análise composicional da pupa do bicho-da-seda (Bombyx mori L.)}

\author{
CONNIE MITCHELLE GRISALES-MUÑOZ¹, FREDY JAVIER LOPEZ-MOLINA²
}

Historial del Artículo

Recibido para evaluación: 1 de Enero 2019.

Aprobado para publicación: 1 de Mayo 2020.

* Proyecto de investigación de orígen: "Desarrollo Tecnológico para la obtención de productos orgánicos e innovadores de seda natural". Financiación. Sistema General de Regalías. Culminación: diciembre de 2020.

1 Universidad del Cauca, Facultad de Ciencias Agrarias, Grupo de Investigación Sistemas Integrados de Producción Agropecuaria, Forestal y Acuícola (SISINPRO). Ingeniera Agropecuaria. Popayán, Colombia. Orcid: http://orcid.org/0000-0003-4702-0706

2 Universidad del Cauca, Departamento de Ciencias Agropecuarias, Facultad de Ciencias Agrarias, Grupo de Investigación Sistemas Integrados de Producción Agropecuaria, Forestal y Acuícola (SISINPRO). M Sc. Ciencias Agrarias. Popayán, Colombia. Orcid: http://orcid.org/0000-0002-4414-1417

Correspondencia: conniegrisales@unicauca.edu.co

Cómo citar este artículo: CONNIE MITCHELLE GRISALES-MUÑOZ, FREDY JAVIER LOPEZMOLINA. Análisis composicional de la pupa de gusano de seda (Bombyx mori L.). Revista Biotecnología en el Sector Agropecuario y Agroindustrial,18(2),2020.126-134, DOI:http:// dx.doi.org/10.18684/BSAA(18)126-134 


\section{RESUMEN}

La sericultura en el Departamento del Cauca se encuentra en una dinámica constante que busca fortalecer distintos eslabones de su cadena productiva. En el proceso de devanado del capullo se genera la pupa de gusano de seda como subproducto, el cual no es aprovechado de manera eficiente. Con el objeto de evaluar la pupa y harina de gusano de seda, como fuentes alternativas de inclusión en dietas para la nutrición animal, se realizó el análisis composicional de materia seca (\%MSA), humedad (\%HR), cenizas (\%CNZ), proteína bruta (\%PB), extracto etéreo (\%EE), quitina (\%QTN) y la digestibilidad in vitro de la materia seca (\%DivMS). Para ello, se utilizaron pupas frescas (PGS) y harina de pupa (PGSh), suministradas por sericultores asociados a CORSEDA. Los resultados efectuados en base seca arrojaron valores de PB de 50,05 y $51,73 \%$; EE 41,36 y $37,62 \%$; QTN 2,47 y $5,49 \%$, para pupa y harina respectivamente. En términos de la digestibilidad de la materia seca, la pupa tuvo un mejor comportamiento con 84,73 contra $77,45 \%$ respecto a la harina. El perfil nutricional de la pupa fresca y la harina permitió determinar que son materias primas potenciales para ser utilizadas en la formulación de balanceados para la industria animal.

\section{ABSTRACT}

The sericulture in Cauca department is a constant dynamic looking for strengthening different tiers of its productive chain. In the process of winding the cocoon, the pupa of the silkworm is generated as a by-product, which has not been accurately exploited. In order to evaluate the pupa and silkworm meal, as alternative sources to include in diets for animal nutrition, the compositional analysis was made from dry matter (\%MSA), humidity (\%HR), ash (\%CNZ), crude protein (\%PB), ether extract (\%EE), chitin (\%QTN) and in vitro digestibility of dry matter (\% DivMS). For this purpose, we used fresh pupae (PGS) and pupal meal (PGSh), supplied by CORSEDA's farmers. The dry base results showed values of PB 50,05 and 51,73\%; EE 41,36 and 37,62\%; QTN 2,47 and 5,49\%, for pupa and meal respectively. In terms of the digestibility of the dry matter, the pupa had a better performance with 84,73 against $77,45 \%$ corresponding to meal. The nutritional profile of the fresh pupa and meal allowed to determine that they are potential raw materials to be used in the formulation of balanced food for the animal industry.

\section{RESUMO}

A sericultura no Departamento de Cauca, está em constante dinâmica em busca do fortalecimento de diferentes elos de sua cadeia produtiva. No processo de enrolamento do casulo, a pupa do bicho-da-seda é gerada como um subproduto, que não é usado de forma eficiente. A fim de avaliar a pupa de bicho da seda e farinha, como fontes alternativas para inclusão em dietas de alimentos para animais, a análise da composição foi realizada: matéria seca (\%MSA), humidade (\%HR), cinzas (\%CNZ), proteína bruta (\%PB), extrato etéreo (\%EE), quitina (\%QTN) e digestibilidade in vitro da matéria seca (\%DivMS). Para este propósito, pupas frescas (PGS) e farelo de pupa (PGSh) foram usadas, for-

\section{PALABRAS CLAVE:}

Insectos útiles, Composición química, Nutrientes, Harina animal.

\section{KEYWORDS:}

Useful insects, Chemical composition, Nutrients, Animal flour.

\section{PALAVRAS CHAVE:}

Insetos uteis, Composição química, Nutrientes, Farinha animal. 
necidas pelos agricultores da CORSEDA. Os resultados da base seca mostraram valores de PB de 50,05 e 51,73\%; EE 41,36 e 37,62\%; QTN 2,47 e 5,49\%, para pupa e farinha, respectivamente. Em termos de digestibilidade da matéria seca, a pupa teve um melhor desempenho com 84,73 contra $77,45 \%$ com relação à farinha. O perfil nutricional das pupas e farinhas frescas, permite determinar que são matérias-primas potenciais, a serem utilizadas na formulação de balanceamento para a indústria animal.

\section{INTRODUCCIÓN}

Debido a su gran adaptabilidad y amplia distribución, la clase insecta conforma la mayor diversidad y biomasa del planeta, constituyendo cuatro quintas partes del reino animal [1].

Los insectos poseen un alto valor nutritivo, representado principalmente por una fuente de excelente calidad de proteínas y vitaminas, además de que generan gran cantidad de beneficios de carácter ambiental y sanitario, por lo que se consideran como una materia prima alternativa para la industria alimentaria humana y animal $[2,3]$, sin embargo, poco se ha promovido su uso para este fin [1].

En este sentido, la harina de insectos podría reemplazar potencialmente entre el 25 y el $100 \%$ de la harina de soya o de pescado en alimentos para animales [4]; cinco especies de insectos son las más utilizadas en alimentación animal: la mosca soldado negro, gusano de la harina, grillos, gusanos de mosca doméstica y gusanos de seda; esta preferencia se debe a que los buenos niveles de aminoácidos esenciales presentes en las harinas de insectos, con contenidos más altos de como lisina, metionina y leucina, comparados con la harina de soya y de pescado. Son también una buena fuente de ácidos grasos, proporcionando un equilibrio nutricional entre proteína y energía para la alimentación de animales de granja [4, 5].

La sericultura en Colombia se enmarca en tres componentes básicos de la cadena, que comprenden la fase agrícola con el cultivo de morera Morus sp, una fase pecuaria con la cría del gusano de seda Bombyx mori L. para la producción de capullos a partir de los cuales se obtiene la seda, y una fase artesanal o agroindustrial para la obtención de filamentos, tejidos y prendas [6].
La pupa de gusano de seda es uno de los principales subproductos generados en la cadena serícola. Su uso ha sido investigado desde 1926 en áreas del conocimiento como la genética, bioquímica, biología molecular, ciencias agrícolas, biológicas y medicina. Los países con mayor participación en la investigación pertenecen al continente asiático, destacándose Japón, China e India, mientras que el aprovechamiento industrial es de amplio espectro en la industria farmacéutica, médica, cosmética y como una herramienta biotecnológica, y potencialmente en la alimentación humana y animal [7].

Debido al incremento en la demanda de proteína de origen animal, aunado al incremento en los costos de la alimentación y a los problemas relacionados con el abastecimiento de materias primas de calidad para la formulación de alimentos balanceados, se hace necesario investigar alternativas de alimentación viables que puedan suplir la escasez de recursos alimenticios. En este sentido, el objetivo del estudio fue determinar la calidad composicional de la pupa fresca y de la harina, en términos de su análisis proximal, digestibilidad in vitro, porcentaje de quitina y nivel de energía, que permita conocer la proporción de los elementos y macromoléculas que los integran y, con ello, inferir su uso potencial en la alimentación animal.

\section{MÉTODO}

\section{Localización}

La investigación se desarrolló en las instalaciones de la Facultad de Ciencias Agrarias de la Universidad del Cauca (Colombia), a una altura de 1733 msnm, temperatura media de $19^{\circ} \mathrm{C}$ y precipitación de 1.800 $\mathrm{mm}$ anuales.

\section{Material experimental}

Se utilizaron pupas frescas obtenidas después del devanado del capullo de seda, con un peso promedio de $2 \mathrm{~g}$ y $5 \mathrm{~cm}$ de longitud, y harina de pupa con $8 \%$ de humedad, previamente procesada y almacenada al vacío durante un periodo aproximado de 6 meses. Las pupas fueron suministradas por CORSEDA (Corporación para el Desarrollo de la Sericultura del Cauca) provenientes de diferentes unidades de producción serícolas del departamento. 


\section{Obtención de la pupa fresca (PGS)}

Una vez se sometió el capullo al proceso de devanado para obtener la seda, quedó como remanente el forro de la pupa (21 a $23 \%$ de seda no devanable), el cual, para ser removido sin alterar su estructura física, se sometió a un proceso de cocción en agua a $90^{\circ} \mathrm{C}$ durante $45 \mathrm{~min}$, lo que facilitó el rompimiento del hilo permitiendo la liberación de la pupa. Para garantizar su conservación, se sometieron a temperaturas de congelación $\left(-25^{\circ} \mathrm{C}\right)$.

\section{Obtención de la harina de pupa de gusano de seda (PGSh)}

Para la fabricación de la harina, las pupas seleccionadas (20 kg) se secaron en un horno deshidratador de alimentos con flujo de aire mixto a $65^{\circ} \mathrm{C}$ durante $5 \mathrm{~h}$, para conseguir una humedad final entre $8 \mathrm{y}$ $10 \%$ que permitió triturarlas en un molino de tornillo eléctrico. Finalmente, la harina resultante fue empacada al vacío y almacenada durante 6 meses a una temperatura de $-25^{\circ} \mathrm{C}$.

\section{Análisis de laboratorio}

Se seleccionó una muestra representativa de 200 $\mathrm{g}$ de pupa (PGS) y harina (PGSh), respectivamente, para su análisis químico y con ello determinar la calidad nutricional de esta materia prima. En el cuadro 1 se muestran los métodos empleados para determinar la composición nutricional en base seca de la pupa y la harina [8], los cuales se efectuaron por duplicado en el Laboratorio de Nutrición Animal de la Universidad Nacional de Colombia sede Palmira. La energía se determinó con respecto al aporte calórico de la proteína bruta, extracto etéreo y carbohidratos [9], utilizando la siguiente expresión:
La determinación del porcentaje de quitina se realizó químicamente por digestión ácida y alcalina, para finalmente aplicar la expresión 2:

$\%$ QTN $=\frac{\text { masa final }}{\text { masa inicial }} * \frac{\text { muestra inicial }}{\% \text { MS analítica }} * 100$

Con respecto a la digestibilidad in vitro de la materia seca para monogástricos, se aplicó la expresión 3:

$\%$ DivMS $=\frac{(\text { peso muestra }-M S) \text { residuos }}{\text { peso muestra }-M S} * 100$ (Ec. 3)

\section{RESULTADOS}

\section{Análisis composicional}

En el cuadro 2 se muestran los resultados promedio del análisis composicional de la pupa y la harina, en donde se evidencian diferencias significativas $(p<0,05)$ en el contenido de nutrientes presentes en las dos muestras comparadas, en tanto que materia seca y cenizas no se presentaron diferencias.

Proteína. Con respecto a los valores de proteína bruta, se observa que superan el $50 \%$, con valores de 51,73 y $50,05 \%$ para la harina y pupa fresca, respectivamente. En términos del valor promedio de este nutriente, otros estudios reportan valores de proteína cruda para B. mori de 49,3 [6] y $51,1 \%$ [10], similares a lo obtenido en este ensayo, y superiores a lo reportado por Rodríguez et al. [1], con valores de $46,87 \%$ de proteína verdadera. Con respecto a otras especies, estudios reportan niveles de proteína cruda que oscilan entre 55 y 70\% en base seca para 11 insectos comestibles en diferentes localidades de México [11].

$E B(\mathrm{kcal} / \mathrm{kg})=5,66 * P B+9,43 * E E+4,1 *$ CHOS (Ec. 1)

Cuadro 1. Métodos empleados para el análisis químico y composicional de la pupa (PGS) y harina (PGSh).

\begin{tabular}{|l|l|l|}
\hline \multicolumn{2}{|c|}{ Método } & \multicolumn{1}{c|}{ Referencia } \\
\hline Materia seca & Secado de la materia prima a $105^{\circ} \mathrm{C}$ durante $24 \mathrm{~h}$ & AOAC, 1990 \\
\hline Cenizas & Calcinación en mufla a $600^{\circ} \mathrm{C}$ durante $24 \mathrm{~h}$ & AOAC, 1990 \\
\hline Proteína & Método Kjeldahl & Kjeldahl, 1983 \\
\hline Extracto etéreo & Método SOXHLET & AOAC, 1990 \\
\hline Quitina & Método químico (digestión acida y alcalina) & Van Soest \& Wine, 1975 \\
\hline Extracto libre de nitrógeno & Método matemático por diferencia & \\
\hline Energía bruta & Método matemático & \\
\hline Digestibilidad in vitro & Método Pre-cecal & Parr, 1941 \\
\hline
\end{tabular}


Cuadro 2. Análisis proximal y significancia estadística, de la pupa (PGS) y la harina (PGSh), de gusano de seda Bombyx mori L, en base seca.

\begin{tabular}{|l|l|l|l|l|l|}
\hline \multicolumn{1}{|c|}{ Análisis (\%) } & \multicolumn{1}{|c|}{ Base Seca \% (PGS) } & \multicolumn{1}{c|}{ DS } & \multicolumn{1}{c|}{ BaseSeca\% (PGSh) } & \multicolumn{1}{c|}{ DS } & \multicolumn{1}{c|}{ Sig (p< 0,05)* } \\
\hline Materia Seca & 95,13 & 1,520 & 94,4 & 0,077 & 0,563 \\
\hline Proteína Cruda & 50,05 & 0,445 & 51,73 & 0,261 & 0,044 \\
\hline Extracto Etéreo & 41,36 & 0,127 & 37,62 & 0,657 & 0,016 \\
\hline Quitina & 2,47 & 0,388 & 5,49 & 0,261 & 0,012 \\
\hline Cenizas & 3,42 & 0,091 & 3,36 & 0,014 & 0,491 \\
\hline Extracto Libre de Nitrógeno & 2,7 & 0,162 & 1,8 & 0,120 & 0,025 \\
\hline
\end{tabular}

DS; Desviación Estándar.

*La probabilidad del estadístico es inferior a 0,05, por lo que se puede inferir, que existen diferencias en todos los componentes a excepción de la materia seca y cenizas.

En términos del grado de digestibilidad de proteína en pepsina al $0,2 \%$, se reporta un valor de $79,46 \%$, lo cual permite inferir su alto potencial para el uso en la alimentación, tanto animal como humana $[1,10]$.

En este análisis no se realizó el perfil de aminoácidos presentes, sin embargo, se resaltan investigaciones $[7,10]$ que reportan la presencia de 18 aminoácidos, de los cuales sobresalen el ácido glutámico y aspártico con un valor mayor a $10 \mathrm{~g} / 100 \mathrm{~g}$ de proteína; siguen en orden de importancia la arginina, leucina, tirosina, fenilalanina, lisina, alanina y valina, con contenidos entre 10 y $5 \mathrm{~g} / 100 \mathrm{~g}$; la threonina, serina, prolina, glicina, histidina, metionina e isoleucina que están presentes entre 1 y $5 \mathrm{~g}$ en $100 \mathrm{~g}$, mientras que el ácido cistéico y cisteína, están presenten en menos de un $1 \mathrm{~g}$ sobre $100 \mathrm{~g}$ de la proteína [6].

En este contexto, los aminoácidos se clasifican como esenciales y no esenciales, siendo los primeros los que más participan en la construcción de proteína muscular y por lo tanto a ganancia de peso en la mayoría de los animales [12]. En particular, las especies menores requieren de los 8 aminoácidos esenciales (Isoleucina, Leucina, Lisina, Metionina, Triptófano, Fenilalanina, Treonina y Valina) [13], los cuales son condicionantes en la mayoría de los procesos metabólicos y se traducen en mejoras de los parámetros productivos en los animales.

La pupa de gusano de seda presentó valores de 4,16, $9,22,5,32,3,20,0,5,50,4,61$ y 6,23 de los aminoácidos mencionados anteriormente, sobre el $100 \%$ de la proteína. Contrastando estos valores con otras materias primas de uso común en la industria alimentaria, como la harina de pescado (54\% PB) que contiene $3,81,6,45,6,10,2,36,0,81,3,75,4,03$ y 4,74 respectivamente [14], se puede observar el potencial de este material para el uso en dietas para la alimentación animal. Otras investigaciones concluyeron que, en el caso particular de los pollos de engorde, se requieren altas cantidades de aminoácidos, en especial lisina y metionina, de lo contrario sus parámetros productivos se verán seriamente afectados. Para suplir esta exigencia nutricional, los balanceados para animales requieren utilizar materias primas de origen animal y vegetal con alta cantidad de aminoácidos, lo que permitiría en cierta medida, hacer un aporte importante de estos nutrimentos [13]; sin embargo, los altos costos de producción, debido principalmente a la dependencia de materias primas proteicas importadas para la producción de alimentos balanceados como la harina de pescado, hacen que las industrias pecuarias se vean seriamente afectadas en su rentabilidad [15]. En este contexto, son varios los autores $[16,17]$ que tratan de identificar subproductos industriales de origen animal, como los ensilados de residuos pesqueros en alimentación de especies menores, con el objeto de encontrar alternativas de alimentación no convencional, que den un uso eficiente a los subproductos generados y que, a su vez, permitan en cierta medida, disminuir los costos de producción.

Extracto etéreo. Se encontró que la pupa tiene mayor contenido de grasas $(41,36 \%)$ que la harina $(37,62 \%)$. En este aspecto, se han encontrado reportes inferiores de 20,63 y $26,99 \%$ para pupa [1, 7] y 16,3 y $34,4 \%$ para larva de B. mori, respectivamente [1].

Las grasas presentes en la harina de pupa representan más de la tercera parte de la composición química total, lo que denota la calidad de esta materia prima como fuente energética. En efecto, estudios [1, 17, 18] reportan que los insectos contienen una mayor cantidad de ácidos mono insaturados y poliinsaturados. 
Si se analiza la relación entre el contenido proteínico y el perfil lipídico, en especial la concentración de ácidos insaturados, resulta valioso el uso potencial de la harina en la fabricación de balanceados, ya que se favorece la relación energética/proteica, permitiendo que el animal emplee los lípidos como fuente de energía sin generar gastos proteicos innecesarios, que aumentarían los costos de producción y contaminación por la formación de exudados nitrogenados [19].

Por otra parte, son varios los estudios que tratan de evaluar diferentes alternativas nutricionales en alimentación de especies menores, buscando fuentes energéticas y proteicas de bajo costo o disponibles en la región, que permitan disminuir los costos de producción sin que se afecten los parámetros productivos [20,21].

Quitina. En relación a los carbohidratos estructurales, la quitina y la fibra cruda, componen la estructura externa o exoesqueleto de los insectos, formando un complejo polisacárido de alto peso molecular [22, 23].

En términos de los valores presentes en la cutícula de la pupa, se evidencia un contenido de quitina en la harina y pupa de 5,49 y $2,47 \%$, respectivamente. Por otra parte, en determinaciones anteriores independientes en muestras de harina de pupa, se encontraron resultados de fibra cruda de 2,41 y $2,72 \%$.

El contenido de fibra en la harina es menor al 3\%, lo que favorece su utilización en raciones animales, ya que una alta concentración de fibra en piensos para nutrición animal en monogástricos, produce un incremento en el consumo para adquirir los niveles de energía digerible necesaria para el crecimiento y mantenimiento [24] y, a la vez, puede afectar parámetros de digestibilidad en la dieta [25].

Cenizas. Los valores de cenizas son similares para ambas muestras, encontrando valores de 3,36 y $3,42 \%$ entre harina y pupa respectivamente. Los minerales son esenciales para desarrollar diversas funciones en el organismo, actuando como catalizadores de reacciones metabólicas; este contenido debe tenerse en cuenta cuando se pretende dar un aprovechamiento a la especie, como suplemento en la fortificación de piensos para consumo animal [26] y en alimentación humana [27].

Energía. El balance óptimo de energía y proteína en la dieta es importante, ya que un exceso o deficiencia genera un retraso en la tasa de crecimiento. Si hay deficiencias energéticas, el organismo hará uso de la proteína, disminuyendo el aporte de este nutriente para la síntesis de tejidos. Por el contrario, un exceso de energía genera que el animal sacie su apetito, antes de ingerir una cantidad suficiente de proteína para satisfacer la tasa de crecimiento [28].

En estudios realizados anteriormente con bomba calorimétrica en muestras de pupa de gusano de seda, en los laboratorios Aval Químico y en el de Bromatología y Abonos Orgánicos de la Universidad de Nariño, se obtuvieron valores de 6890 y $6920 \mathrm{kcal} / \mathrm{kg}$, respectivamente, los cuales son superiores al realizado por Rodríguez et al. [1] que obtuvieron $6.220 \mathrm{kcal} / \mathrm{kg}$ de energía bruta; con respecto a esta investigación, los valores calculados por método matemático arrojaron 6.846 y $6.551 \mathrm{kcal} / \mathrm{kg}$ para pupa y harina, respectivamente.

En este sentido, la pupa de gusano de seda posee mayor cantidad de energía bruta que otras materias primas proteicas utilizadas en la nutrición animal, como la harina de carne $(3.988 \mathrm{kcal} / \mathrm{kg})$, de pescado $(4.040$ $\mathrm{kcal} / \mathrm{kg}$ ), de plumas $(5.137 \mathrm{kcal} / \mathrm{kg})$, de vísceras (5.219 $\mathrm{kcal} / \mathrm{kg}$, de sangre $(5.103 \mathrm{kcal} / \mathrm{kg})$, de soya $(4.471$ $\mathrm{kcal} / \mathrm{kg}$ ) y materias de carácter energético como el gluten de trigo $(5.123 \mathrm{kcal} / \mathrm{kg})$ y de maíz $(4.977 \mathrm{kcal} /$ kg) [29]. Estas diferencias están dadas principalmente por la alta concentración de grasas presentes en la muestra estudiada, que le brindan un valor energético interesante para la formulación de piensos.

Digestibilidad in vitro. En la medición de este parámetro, se evaluaron tres muestras de cada material y el resultado promedio se reporta en la figura 1.

Se ha planteado la hipótesis de que la matriz de la quitina puede reducir el acceso de quitinasas o proteinasas a sus sustratos, reduciendo así no sólo su digestibilidad, sino también la de los lípidos y proteínas. Esto trae como consecuencia una baja utilización de nutrientes y del rendimiento en el crecimiento de los animales alimentados con esta materia prima [28].

Existen estudios que reportan que los insectos de cuerpo blando como el gusano de seda, contienen menos quitina y son más digestibles que otros [4, 22, 23]. En este estudio se encontró que la digestibilidad de la harina fue menor (77,45\% en promedio), debido a la cantidad de carbohidrato estructural representado en el $5,49 \%$ de quitina presente en la muestra; en contraste, en la pupa los resultados de digestibilidad son más altos 
Figura 1. Promedios de digestibilidad In Vitro de la materia seca, de la harina y pupa de gusano de seda.

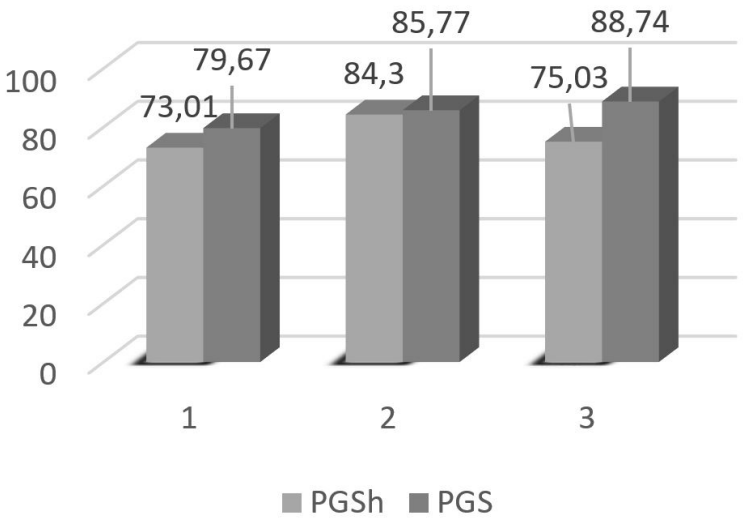

$(84,73 \%)$, con un contenido menor de quitina (2,47\%). Si se comparan estos resultados con la harina de pescado, ésta presenta valores de digestibilidad entre 80 a $90 \%$, dependiendo de la fuente utilizada, del valor proteico inicial y del método de fabricación [30].

\section{CONCLUSIONES}

En Colombia no existen reportes del aprovechamiento de la pupa de B. mori como alternativa para la alimentación animal, ya que la cadena serícola es valorada principalmente para la producción artesanal o agroindustrial de fibras textiles; sin embargo, de acuerdo con los resultados reportados en esta investigación, puede ser utilizada para la alimentación por su alto contenido proteico y energético, características que pueden convertirla en un ingrediente con un alto valor agregado en la industria de los alimentos balanceados.

Las diferencias en contenido de la mayoría de los nutrientes de la harina y la pupa no son significativas, pero es interesante resaltar una mayor presencia de extracto etéreo en la pupa $(41,36)$ que en la harina $(37,62)$, lo que permite inferir que la transformación de la pupa en harina, buscando generar un mayor tiempo de conservación de esta materia prima, es una opción que no altera de manera significativa el perfil de nutrientes y su posterior uso como alimento.

Las proporciones de quitina encontradas influyen directamente sobre la digestibilidad, siendo mayor en la pupa $(84,73 \%)$ que en la harina $(77,45 \%)$.
Las características nutricionales presentes hacen que el gusano de seda B. mori, ya sea como harina o pupa fresca, pueden ser considerado como una alternativa alimentaria de alto valor biológico, representado principalmente en un buen balance entre el aporte proteico y lipídico.

\section{AGRADECIMIENTOS}

Los autores agradecen a CORSEDA por el aporte de la pupa como materia prima; al Laboratorio de Nutrición Animal de la Universidad Nacional de Colombia Sede Palmira, por la prestación de sus equipos y personal capacitado; al proyecto "Desarrollo tecnológico para la obtención de productos orgánicos e innovadores de seda natural", convenio entre la Gobernación del Cauca, Universidad del Cauca y Sistema General del Regalías.

\section{REFERENCIAS}

[1] RODRÍGUEZ, A., PINO, J., ÁNGELES, S., GARCÍA, BARRÓN, A. y CALLEJAS, J. Valor nutritivo de larvas y pupas de gusano de seda (Bombyx mori L.) (Lepidoptera: Bombycidae). Revista Colombiana de Entomología, 42(1), 2016, p. 69-74.

[2] ITALIA. ORGANIZACIÓN DE LAS NACIONES UNIDAS PARA LA AGRICULTURA Y LA ALIMENTACIÓN (FAO). La contribución de los insectos a la seguridad alimentaria, los medios de vida y el medio ambiente ¿Qué es La entomología?. Roma (Italia): I, 2016.

[3] ITALIA. ORGANIZACIÓN DE LAS NACIONES UNIDAS PARA LAAGRICULTURA Y LAALIMENTACIÓN (FAO). Insectos para alimentación y piensos. 2018. Disponible: http://www.fao.org/edible-insects/en/. [citado 9 octubre de 2018].

[4] HENRY, M., GASCO, L., PICCOLO, G. and FOUNTOULAKI, E. Review on the use of insects in the diet of farmed fish: Past and future. Animal Feed Sciences \& Technologies, 203(1), 2016, p. 1-22. doi: 10.1016/j.anifeedsci.2015.03.001

[5] MAKKAR, H., TRAN, G., HEUZÉ, V. y ANKERS, P. Uso de insectos como alimento para animales. Animal Feed Sciences \& Technologies, 197, 2014 p. 1-33. doi: 10.1016/j.anifeedsci.2014.07.008

[6] RODRÍGUEZ, A., MONTER, A., MAZA, A., MARTíNEZ, J., EHSAN, M. y LARA, F. Manual de Sericultura 
en Hidalgo: Principios básicos. Hidalgo (México): UPFIM, CONACYT, FOMIX, COCYTEH, 2012.

[7] ANIMANATURALIS. Seda: historia, producción, y alternativas. Anima Naturalis Internacional. 2018. Disponible: https://www.animanaturalis. $\mathrm{org} / \mathrm{p} / \mathrm{seda}$ historia-produccion-y-alternativas [citado 9 octubre de 2018].

[8] LETERME, P. y ESTRADA, F. Análisis de alimentos y forrajes: protocolos de laboratorio. Palmira (Colombia): 2016.

[9] AIRAHUACHO, F. y VERGARA, V. Evaluación de dos niveles de energía digestible en base a los estándares nutricionales del NRC (1995) en dietas de crecimiento para cuyes (Cavia porcellus L.). Revista de investigación Veterinaria, 28(2), 2017, p. 255 - 264. doi: http://dx.doi.org/10.15381/rivep. v28i2.13079

[10] PEREIRA, N., FERRARESE, M., MATSUSHITA, O. and DE SOUZA, N. Proximate composition and fatty acid profile of Bombyx mori L. chrysalis toast. Journal of Food Compositionals Analysis, 16(4), 2003, p. 451-457. doi: https://doi.org/10.1016/ S0889-1575(03)00016-4

[11] ROSTRO, B., QUINTERO, B., RAMOS, J., PINO, J.M., ÁNGELES, S., GARCÍA, A. y BARRERA, D. Análisis químico y nutricional de tres insectos comestibles de interés comercial en la zona arqueológica del municipio de San Juan Teotihuacán y en Otumba, en el estado de México. Interciencia, 37(12), 2012, p. 914-920.

[12] MORALES, W., RODRÍGUEZ, V. y VERJAN, N. Parámetros productivos y económicos de gallinas ponedoras ISA Brown en segundo ciclo de producción suplementadas con aminoácidos no esenciales. Revista de Investigaciones Veterinarias del Perú, 29(2), 2018, p. 533. doi: http://dx.doi. org/10.15381/rivep.v29i2.14481

[13] CHAVEZ, M., BERNAL, J., TÁMBARA, Y., PÉREZ, E. and SÁNCHEZ, A. Essential aminoacid contents of protein hydrolized supplements used in the diet of laying hens. Revista de Producción Animal, 29(2), 2017, p. 73-76.

[14] ROSTAGNO, H., TEIXEIRA, L.F., LOPES, J., GOMES, P.C., FLÁVIA DE OLIVEIRA, R., CLEMENTINO, D., SOARES, A. e DE TOLEDO, S.L.. Tablas brasileñas para aves y cerdos: composición de alimentos y requerimientos nutricionales. 3 ed. Vicosa (Brasil): Universidade Federal de Viçosa, 2011, 259 p.

[15] PEREA, C., GARCÉS, Y., MUÑOZ, L., HOYOS, J. y GÓMEZ, J. Valoración económica del uso de ensilaje de residuos piscícolas en la alimentación de Oreochromis spp. Biotecnología en el Sector Agropecuario y Agroindustrial, 16(1), 2018, p. 43-51. doi: http://dx.doi.org/10.18684/bsaa.v16n1.623

[16] GÓMEZ, G., ORTIZ, M., PEREA, C. and LOPEZ, F. Evaluación del ensilaje de vísceras de tilapia roja (Oreochromis spp) en alimentación de pollos de engorde. Biotecnología en el Sector Agropecuario y Agroindustrial, 12(1), 2014, p. 106-114.

[17] PINO, M., RAMOS-ELORDUY, J. y MEDEIROS, C. Los insectos comestibles comercializados en los mercados de Cuautitlán de Romero Rubio, Estado de México, México. Sitientibus Serie. Ciencias Biológicas (Etnobiología), 6, 2006, p. 58-64.

[18] VAN HUIS, A., VAN ITTERBEECK, J., KLUNDER, H., MERTENS, E., HALLORAN, A., MUIR, G. and VANTOMME, P. Edible insects future prospects for food and feed security. Rome (Italy): Wageningen UR FAO Forestry Paper, FAO, 2013, 171, ISSN 0258-6150.

[19] CHEN, H., TIAN, J., WANG, Y, YANG, K., JI, H. and $\mathrm{LI}, \mathrm{J}$. Effects of dietary soybean oil replacement by silkworm, (Bombyx mori L.), chrysalis oil on growth performance, tissue fatty acid composition, and health status of juvenile jian carp, (Cyprinus carpio) var. Jian. Journal of the World Aquaculture Society, 48(3), 2017, p. 453-466. doi: https://doi. org/10.1111/jwas.12373

[20] SANTURINO, C., GARCÍA, J., SIERRA, P., CASTRO, M., CALVO, M. y FONTECHA, J. Los insectos como complemento nutricional de la dieta: fuente de lípidos potencialmente bioactivos. Revista Alimentación, Nutrición y Salud, 23(2), 2016, p. 50-56.

[21] J, H., ZHANG, J.L., HUANG, J.Q., CHENG, X.F. and LIU, C. Effect of replacement of dietary fish meal with silkworm pupae meal on growth performance, body composition, intestinal protease activity and health status in juvenile Jian carp (Cyprinus carpio var. Jian). Journal of the Aquaculture Research, 46(5), 2015, p. 12091221. doi. https://doi.org/10.1111/are.12276

[22] MONTER, J., TIRADO, J., ZAMUDIO, P., RÍOS, C., ORNELAS, J., SALGADO, R., ESPINOSA, V. y HERNÁNDEZ, F. Extracción y caracterización de propiedades fisicoquímicas, morfológicas y estructurales de quitina y quitosoano de Brachystola magna (Girard). Revista Mexicana de Ingeniería Química, 15(3), 2016, p.749-761.

[23] LÓPEZ, M., GIRALDO, G. y MEJÍA-DORIA, C. Evaluación de la capacidad de la quitina, para atrapar grasa. Agronomía Colombiana, 34(1), 2016, p. 2-4. Doi: 10.15446/agron.colomb 
[24] SALCEDO, L., NÚÑEZ, E., TORRES, O., ARAGADVAY, $M$. and YUNGAN, G. Effects physiopathological of secondary compounds in monogastric: A Review. Journal Selva Andina Animal Science, 4(1), 2017, p. 82-92.

[25] NUÑEZ, O. Los costos de la alimentación en la producción pecuaria. Journal Selva Andina Animal Science, 4(2), 2017, p. 93-94.

[26] ANANKWARE, J., PAARECHUGA, K., OSECKRE, E. and OBENGH, D. Insects as Food and Feed: A Review. International Journal of Agricultural Research and Review, 3(1), 2015, p. 143-151.

[27] CARTAY, R. Between shock and disgust: the consumption of insects in the amazon basin. the case of Rhynchophorus palmarum (Coleoptera Curculionidae). Revista Colombiana de Antropología, 54(2), 2018, p. 143-159. doi: http://dx.doi. org/10.22380/2539472x.465

[28] MARISCAL, G. y RAMIREZ, E. Determinación de la digestibilidad de la proteína, aminoácidos y energía de canola integral en cerdos en crecimiento. Revista Mexicana de Ciencias Pecuarias, 8(3), 2017, p. 297-304. doi: http://dx.doi. org/10.22319/rmcp.v8i3.4505.

[29] FUNDACIÓN ESPAÑOLA PARA EL DESARROLLO DE LA NUTRICIÓN ANIMAL (FEDNA). Tablas de composición y valor nutritivo de alimentos para la fabricación de piensos compuestos. 2010. Disponible https://www.fundacionfedna. org/ingredientes-para-piensos [citado 10, septiembre de 2018].

[30] CALDERON, V., CHURACUTIPA, M., SALAS, A., BARRIGA, M. and ARANIBAR, M. Effect of the inclusion of silage of trout residues in pigs feed and its effect on the productive performance and the taste of meat. Revista de Investigaciones Veterinarias del Perú, 28(2), 2017, p. 265274. doi: 10.15381/rivep.v28i2.13055 